\title{
59. The Tumours of The Skull
}

\author{
Shuji Kamano, Shigeru Kobayashi and Kazuo Takeuchi \\ Division of Neurosurgery, Toranomon Hospital, Tokyo
}

There are 14 cases which originate from, invade or metastate the base of the skull. They have various signs or symptoms of the cranial nerves. Nevertheless establishment of diagnosis is not so difficult, because plain craniogram or tomogram of the skull contribute much for it. Also inspection of the throat and the nasal cavity is sometimes so helpful.

\section{Five Cases of Intracranial Hypertension of Unknown Etiology}

\author{
Kiku Nakao, Shozo Kito, Kunio Matsuda, Nakaaki Osawa, \\ Tatsuo Aral, Hideo Yamamoto, Kimiyoshi Uono \\ and Toshiji Mozal \\ 3rd Department of Internal Med., University of Tokyo School of Medicine
}

Five cases of intracranial hypertension of unknown etiology have been studied. Of five four cases were females. Ages ranged from 13 to 43 years. Head trauma, otologic diseases and endocrinological disorders were not considered to be significant etiologically. Obesity was noted in one case. There were no neurological focal signs in all of the cases and even no subjective symptoms of increased intracranial pressure such as headache, nausea and vomiting were complained in 2 cases and choked discs were discovered by chance. Enlarged ventricles were observed in 2 cases. Quality of cerebrospinal fluid was essentially normal except in one case in which total protein and cell count were increased. Electroencephalograms were abnormal in 3 cases. In 3 cases, choked discs and signs of increased intracranial pressure disappeared spontaneously and fluid pressures also returned to normal. In one case, lumbar punctures also returned to normal. In one case, lumbar punctures were repeated as a symptomatic therapy and thus the pressure increased acceleratedly. Unconsciousness and general emaciation developed and expired after one month of hospitalization. Autopsy revealed the normal brain both macroscopically and histologically except the marked pressure cone. As for another case, syptoms of acute 